Article

\title{
Imparting Photo-responsive Function to Thermo-responsive Iridescent Emulsions
}

\author{
Ryoichi Kondo, Yoshiro Imura, Ke-Hsuan Wang $\mathbb{B}$ and Takeshi Kawai *(i) \\ Department of Industrial Chemistry, Tokyo University of Science, Tokyo 162-0825, Japan; \\ 4218518@ed.tus.ac.jp (R.K.); imura@ci.kagu.tus.ac.jp (Y.I.); wang@ci.kagu.tus.ac.jp (K.-H.W.) \\ * Correspondence: kawai@ci.kagu.tus.ac.jp; Tel.: +81-3-5228-8312
}

Received: 29 September 2018; Accepted: 11 October 2018; Published: 13 October 2018

check for updates

\begin{abstract}
In our previous paper, we reported that thermo-responsive emulsions can be prepared based on a long-chain amidoamine derivative (C18AA) and tetraoctylammonium bromide (TOAB), and that the $\mathrm{C} 18 \mathrm{AA}+\mathrm{TOAB}$ emulsions developed a characteristic interference color in a narrow temperature range. However, the coloration of the original C18AA + TOAB at room temperature exhibited poor brightness. In the present study, we show that the addition of $\mathrm{NaOH}$ is effective in both lowering the coloration temperature and improving the brightness of C18AA + TOAB emulsion considerably. Furthermore, we demonstrate that photo-response function can be imparted to C18AA + TOAB iridescent emulsions by introducing a photochromic naphthopyran derivative (Pyran) that reversibly changes from white to yellow upon UV irradiation. The C18AA + TOAB emulsions containing Pyran shows a dual stimuli-responsive iridescent property, and the emulsion color is controllable and reversible through both UV irradiation and temperature.
\end{abstract}

Keywords: coloring temperature; iridescent emulsions; thermo-response; photo-response

\section{Introduction}

Stimuli-responsive materials have attracted considerable attention because of their potential applications in various fields of material science [1-6]. In particular, stimuli-responsive coloring materials that can switch their color reversibly in response to an external stimulus, such as temperature, light, electric field, or magnetic field have been widely studied [7-19]. For example, Asher et al. [7] fabricated thermally color-tunable diffraction devices by using crystalline colloidal arrays of monodisperse particles made of poly( $N$-isopropylacrylamide) that shows temperature-induced volume-phase transition. The wavelength of light diffracted from colloidal crystal hydrogel films has been reported to be tunable by light or the concentration of ionic species or organic solvents [8].

In a previous study [20], we demonstrated that an emulsion comprising a dual-surfactant system of a long-chain amidoamine derivative (C18AA) (Figure 1a) and tetraoctylammonium bromide (TOAB) (Figure 1b) shows novel thermo-responsive iridescent phenomenon due to phase inversion on heating from an oil/water emulsion to a water/oil emulsion, passing through a lamellar phase that develops an iridescent color. The lamellar phase has a periodic lamellar structure composed of water, toluene, and surfactant layers of C18AA and TOAB (Figure 1c). The coloration is produced by the interference of light at the periodic layered structure. Furthermore, the coloring temperature and the color of the emulsion are independently controllable by adjusting the concentrations of C18AA and TOAB, respectively. The emulsions developed an iridescent color in a specific narrow temperature range $\left(\sim 3^{\circ} \mathrm{C}\right)$, which indicates that the emulsion exhibits thermo-responsive coloring phenomenon; however, the color is not switchable by application of an external stimulus.

To impart the additional stimulus-responsive coloring function to the $\mathrm{C} 18 \mathrm{AA}+\mathrm{TOAB}$ emulsion, we need to synthesize a C18AA or a TOAB derivative bearing a stimulus-responsive functional 
group, such as photochromic and electrochromic groups. However, it is difficult to design a new functional derivative while maintaining the thermo-responsive coloring function. A suitable method is the introduction of an effective compound that triggers a change in the layered structure or the color itself by an external stimulus. However, undesired effects that induce changes in the original thermo-responsive iridescent function (i.e., lowering the iridescent responsive function) are a cause for concern. The choice of the additional compound is thus crucial for imparting the additional stimuli-responsive function.

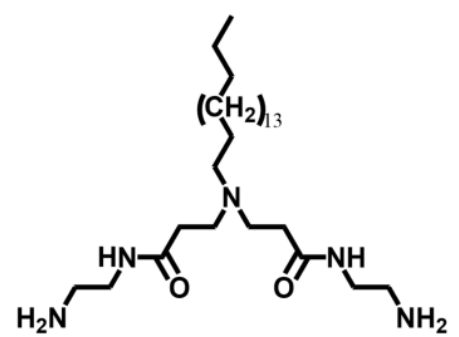

(a)

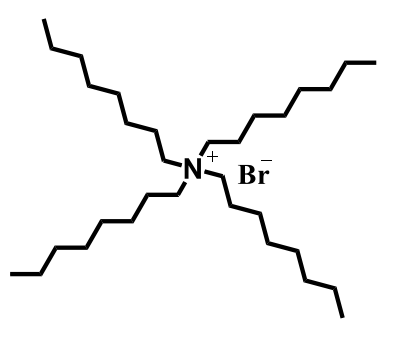

(b)

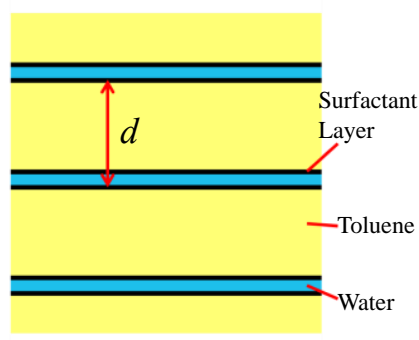

(c)

Figure 1. (a) Molecular structure of C18AA and (b) tetraoctylammonium bromide (TOAB), and (c) schematic illustration of the periodical layered structure of the iridescent emulsion.

The color of photochromic 3,3-diphenyl-3H-naphtho[2,1-b]pyran (Pyran) (Figure 2) changes from white to yellow upon irradiation with UV light because of photoisomerization [21]. Furthermore, as the isomers of Pyran are soluble in toluene, but not in water, we expect that Pyran molecules present in the bulk toluene phase of C18AA + TOAB emulsions are not affected by UV irradiation. This enables us to impart photo-responsive function to the C18AA + TOAB iridescent emulsion without affecting their thermo-responsive iridescent behavior. In this study, we demonstrate that the addition of Pyran in C18AA + TOAB emulsion bestows dual stimuli-responsive (i.e., thermo- and photo-responsive) functions which help in controlling the color of the emulsion in response to both heat and light. Furthermore, we show that the brightness of the iridescent color can be improved by adding $\mathrm{NaOH}$; the original C18AA + TOAB iridescent emulsion at room temperature has poor brightness, which reduces the performance of the dual stimuli-responsive iridescent emulsion.

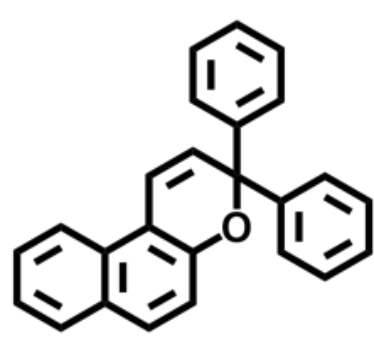

colorless
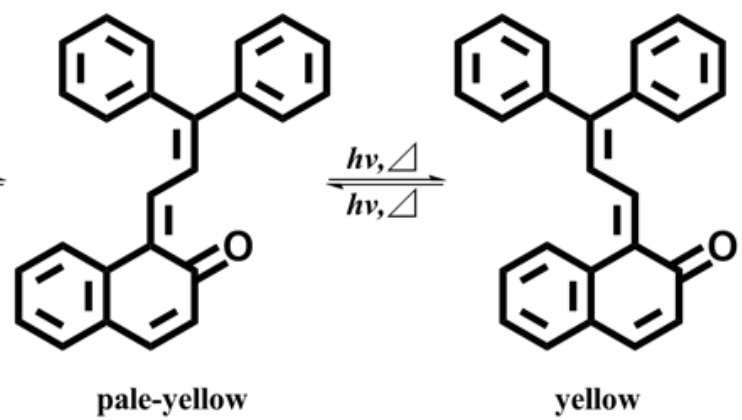

Figure 2. Schematic illustration of photoisomerization of Pyran.

\section{Materials and Methods}

All the chemicals were of reagent grade and obtained from Aldrich (St. Louis, MO. USA), Kanto Chemicals (Tokyo, Japan), or Tokyo Chemical (Tokyo, Japan) Industry. Commercially available reagents and solvents were used without further purification, except methyl acrylate (Kanto Chemicals), which was purified by distillation under reduced pressure in a nitrogen atmosphere, and octadecylamine, which was recrystallized two times from hexane. Pyran (Tokyo Chemical Industry) was used as the 
photochromic compound, and C18AA was synthesized according to the procedure reported in a previous paper $[22,23]$.

A typical iridescent emulsion containing Pyran was prepared as follows. TOAB and Pyran toluene solution $(1.5 \mathrm{~mL})$ was added to an aqueous solution $(0.2 \mathrm{~mL})$ of $\mathrm{C} 18 \mathrm{AA}$ and $\mathrm{NaOH}$, and the mixture was sonicated for $20 \mathrm{~min}$. The total concentrations of $\mathrm{C} 18 \mathrm{AA}, \mathrm{TOAB}, \mathrm{NaOH}$ and Pyran were 30.7, 15, 3 , and $16 \mathrm{mM}$, respectively. The volume fraction of toluene was fixed at 0.88 for all the experiments, whereas the concentrations of $\mathrm{C} 18 \mathrm{AA}, \mathrm{TOAB}, \mathrm{Pyran}$, and $\mathrm{NaOH}$ were varied for preparing various iridescent emulsions.

The direct reflection spectra of C18AA + TOAB emulsions were recorded using a UV-Vis spectrometer (JASCO, V570, Tokyo, Japan) equipped with an absolute reflectivity accessory (JASCO, ARN-475, Tokyo, Japan) at the interface of a quartz cell filled with the emulsion. The temperature of the samples with and without UV irradiation was controlled using a thermostatic water jacket maintained by a refrigerated bath circulator. The lattice spacing of the periodic structure of the iridescent emulsion was calculated using the Bragg equation:

$$
\lambda=2 d\left(n-\sin ^{2} \theta\right)^{1 / 2}
$$

and

$$
\sin ^{2} \theta=-\frac{\lambda^{2}}{4 d^{2}}+n^{2}
$$

where $\lambda$ is the wavelength of the reflection peak, $d$ is the lattice spacing, $\theta$ is the incident and the reflection angle, and $n$ is the average refractive index of the emulsion (Figure 3). The plot of $\lambda^{2}$ against $\sin ^{2} \theta$ was linear, indicating that the iridescent color of the emulsion is definitely derived from the interference of the periodic layered structure of toluene and water phases.

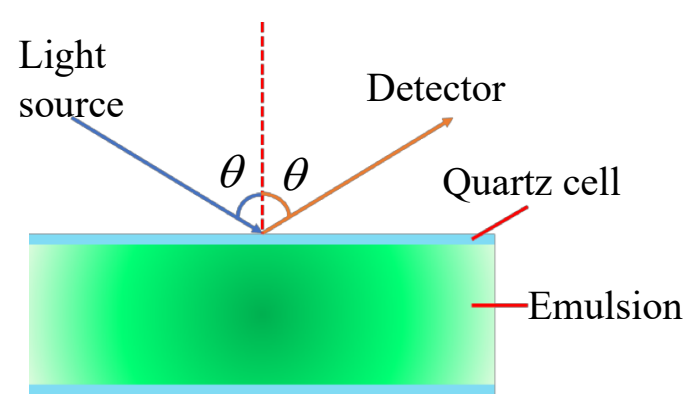

(a)

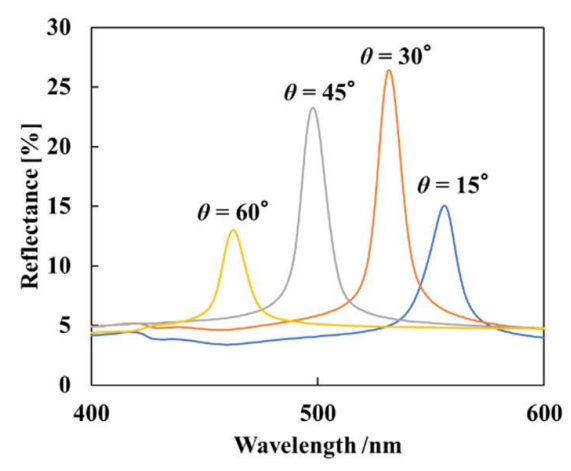

(b)

Figure 3. (a) Schematic illustration of the experimental setup for direct UV-Vis measurements. (b) Direct UV-Vis reflection spectra of the iridescent emulsion at various incident and detection angles.

\section{Results and Discussion}

For investigating the photo-responsive property of the thermo-responsive iridescent emulsion, an emulsion that develops color at room temperature $\left(25^{\circ} \mathrm{C}\right)$ is highly favorable, because the emulsion temperature need not be controlled. In the previous study [20], we demonstrated that the iridescence temperature is controllable in the range of 20 to $50^{\circ} \mathrm{C}$ by tuning the molar ratio of [TOAB] to [C18AA]; however, the emulsions showed a faint iridescence at room temperature (insertion of Figure 4a). As depicted in Figure 4a, the reflectance of the interference peak from the iridescent emulsion of [C18AA] $=30.7 \mathrm{mM}$, which is a measure of iridescent strength, decreases rapidly above $15 \mathrm{mM}$ of TOAB.

The weak coloration of the emulsion deteriorates the dual stimuli-responsiveness. $\mathrm{NaOH}$ was found to be an effective additive for lowering the coloring temperature without losing the brightness 
of the color. Figure $4 \mathrm{~b}$ shows the effect of $\mathrm{NaOH}$ concentration on the coloring temperature and the reflectance of the interference peak of the iridescent emulsions, when the concentrations of C18AA and TOAB were 30.7 and $15 \mathrm{mM}$, respectively. The coloring temperature initially decreased with increase in the $\mathrm{NaOH}$ concentration and then showed no significant variations for $[\mathrm{NaOH}]>5 \mathrm{mM}$. Interestingly, the addition of $\mathrm{NaOH}$ improved the brilliance of the iridescent emulsion. The reflectance increased from $\sim 25$ to $\sim 40 \%$ with the addition of $\mathrm{NaOH}$ and maintained the high value for $[\mathrm{NaOH}]>2 \mathrm{mM}$; however, the reason for the increase in the reflectance is still not understood.

It is completely unclear whether $\mathrm{NaOH}$ affects the interference color, besides the iridescence temperature. As evident from photographs of the iridescent emulsions shown in the insets in Figure 4c, the interference color of bluish green was independent of the concentration of $\mathrm{NaOH}$. This is also confirmed by the finding that the lattice spacing $d$ is almost constant in Figure 4c. Thus, the addition of $\mathrm{NaOH}$ in iridescent emulsions is highly effective in tuning the coloring temperature. In the subsequent experiments, emulsions containing $3 \mathrm{mM} \mathrm{NaOH}$ that colored at room temperature $\left(\sim 25^{\circ} \mathrm{C}\right)$ were used.

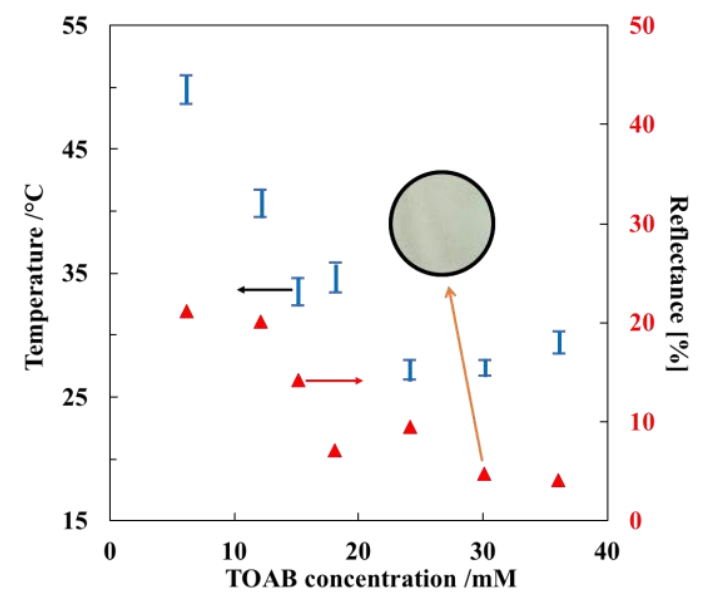

(a)

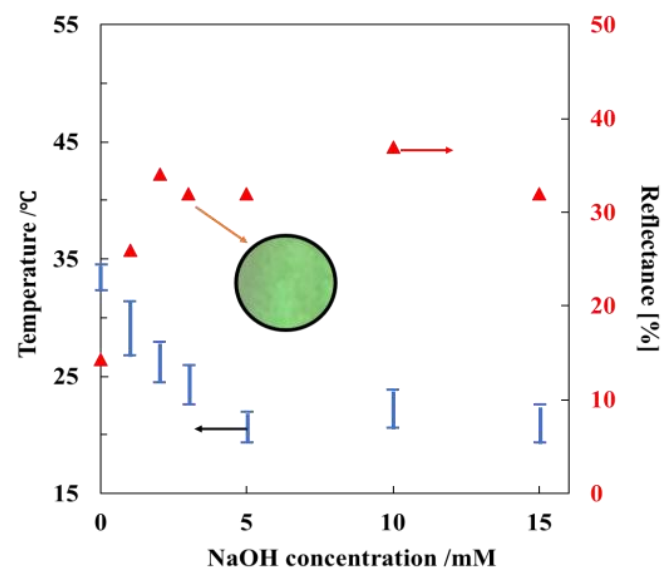

(b)

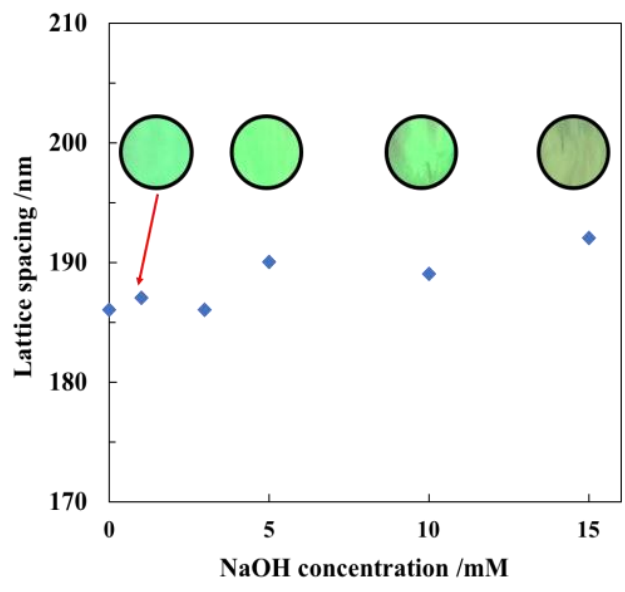

(C)

Figure 4. Effects of (a) TOAB and (b) $\mathrm{NaOH}$ concentration on the coloring temperature range and the reflectance of the interference peak in the UV-Vis reflection spectra of the iridescent emulsion. The incidence and the detection angle of light was $\theta=15^{\circ}$ from the normal. (c) Lattice spacing and photographs of the C18AA + TOAB emulsions vs. $[\mathrm{NaOH}]$, at $[\mathrm{C} 18 \mathrm{AA}]=30.7 \mathrm{mM}$ and $[\mathrm{TOAB}]=15 \mathrm{mM}$.

To impart the additional stimuli function to the thermo-responsive iridescent emulsions, it is important that the additive does not affect the phase inversion behavior and the nature of the 
iridescent emulsions. Photochromic Pyran was used for imparting the photo-responsive function to the thermo-responsive iridescent emulsions, because Pyran is soluble in toluene and photo-isomers have noncharged molecular structures (Figure 2). It is expected that Pyran molecules would be soluble in bulk toluene phase, but would not penetrate at the interface between toluene and water phases where C18AA and TOAB preferentially adsorb to form emulsions. This behavior leads to the conclusion that Pyran does not affect the nature of iridescent emulsions. To confirm this expectation, we examined the effect of addition of Pyran on the coloring temperature range and lattice constant of the emulsions. As shown Figure 5, it is apparent that the characteristic properties are independent of the addition of Pyran. Furthermore, the color of the emulsions with and without Pyran is the same, indicating that Pyran molecules have a very weak interaction with the C18AA + TOAB layers between toluene and water phases.

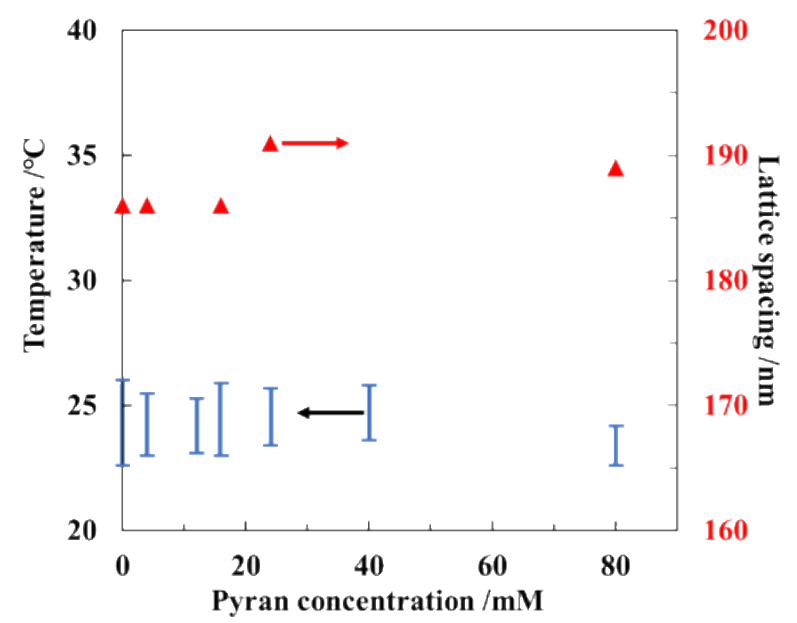

Figure 5. Effect of concentration of Pyran on the coloring temperature range and lattice spacing of the $\mathrm{C} 18 \mathrm{AA}+\mathrm{TOAB}$ emulsions at $[\mathrm{C} 18 \mathrm{AA}]=30.7 \mathrm{mM},[\mathrm{TOAB}]=15 \mathrm{mM}$, and $[\mathrm{NaOH}]=3 \mathrm{mM}$.

The key to impart photo-responsive function to the iridescent emulsions is the photoisomerization behavior of Pyran dissolved in the emulsions. Pyran dissolved in toluene turned yellow upon irradiation with UV light, and the emulsion turned colorless within a few seconds when the UV light was turned off. Similar photo-response time and color change was observed in the C18AA + TOAB emulsions containing Pyran. We thus examined the photo-response of the C18AA + TOAB emulsions under UV irradiation. Figure 6a shows the photographs of the iridescent emulsions containing various concentrations of Pyran with and without UV light irradiation. The color of the emulsion changed from bluish green to green upon UV irradiation; this is reasonable because a combination of yellow and blue gives green. The lattice spacing of the original emulsion and the emulsion under UV irradiation were 185.3 and $184.5 \mathrm{~nm}$, respectively, indicating that the color change upon UV irradiation is derived from the color change of Pyran and not from the change in the interference color. Furthermore, since the color of the emulsions under UV irradiation is independent of the concentration of Pyran, we can reproducibly obtain the combination color of the interference color of the C18AA + TOAB emulsion and the photochromic color of Pyran molecules.

As the phase inversion behavior and the coloring properties of the iridescent emulsions of C18AA were unaffected by the presence of photochromic Pyran, the introduction of Pyran is an effective method to prepare thermo- and photo-responsive iridescent emulsions. Figure $6 \mathrm{~b}$ shows the photographs of the iridescent emulsion under controlling exposure to UV light and temperature. It is apparent that the emulsion color is controllable and reversible through both UV irradiation and temperature. Thus, we have successfully demonstrated that dual stimuli-responsive coloring emulsions can be prepared easily by the introduction of photochromic Pyran in thermo-responsive iridescent emulsions of C18AA. Further, the emulsions developed iridescent colors at least two months. 


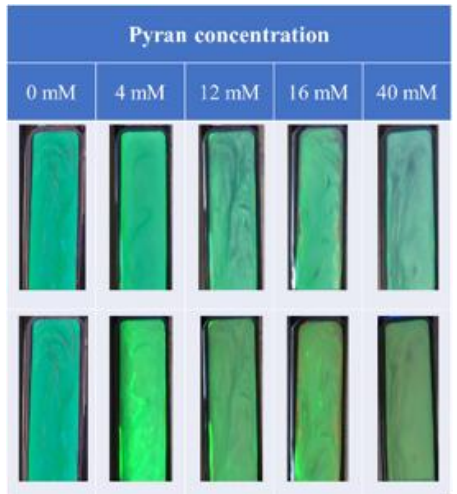

(a)
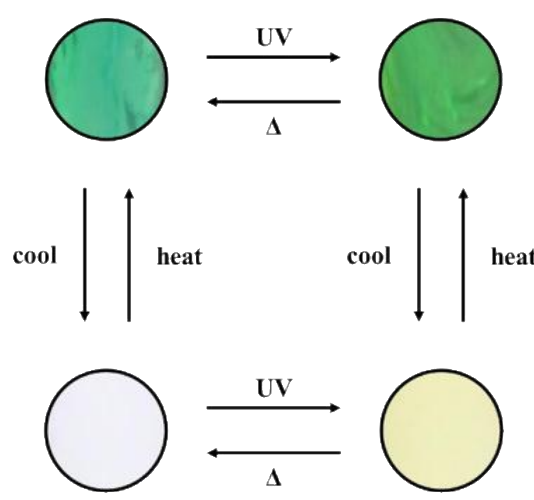

(b)

Figure 6. (a) Photographs of the original (upper) and the UV irradiated (bottom) emulsions at various Pyran concentrations; $[\mathrm{C} 18 \mathrm{AA}]=30.7 \mathrm{mM}$, $[\mathrm{TOAB}]=15 \mathrm{mM}$, and $[\mathrm{NaOH}]=3 \mathrm{mM}$. (b) Color variation of C18AA + TOAB emulsion in response to UV light and heat; [C18AA] $=30.7 \mathrm{mM}$, $[\mathrm{TOAB}]=15 \mathrm{mM}$, $[\mathrm{NaOH}]=3 \mathrm{mM}$, and $[$ Pyran $]=16 \mathrm{mM}$.

Other color changes induced by irradiation of UV light, instead of bluish green to green, are also possible, because the interference color of the iridescent emulsions is controllable by varying the concentration of C18AA [20]. For example, color changes from pink to pale orange and blue to light blue can be accomplished using $29.5 \mathrm{mM}$ and $40 \mathrm{mM} \mathrm{C18AA}$, respectively (Figure 7). Thus, tuning the starting emulsion color enables broadening of the color range of iridescent emulsions easily. However, in the present system, the color variation is limited because the additional color is fixed by the yellow color of Pyran, whereas the original interference color is controllable by the concentration of C18AA. To overcome this limitation and to realize the desired color variation, i.e., changes from one desired color to another desired color, we have to extend the range of the additional color. Such extension is quite possible by introducing water- or toluene-soluble photo-responsive coloring materials, such as photochromic compounds, fluorescent compounds, and quantum dots, into the present iridescent emulsion system.
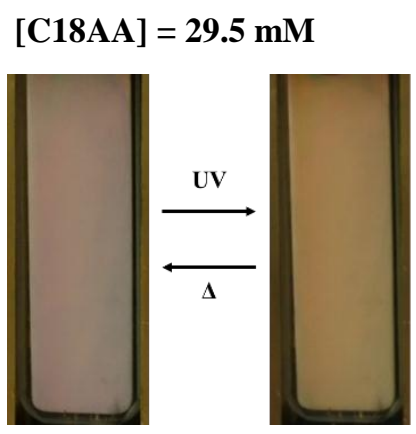

$[\mathrm{C18AA}]=30.7 \mathrm{mM}$

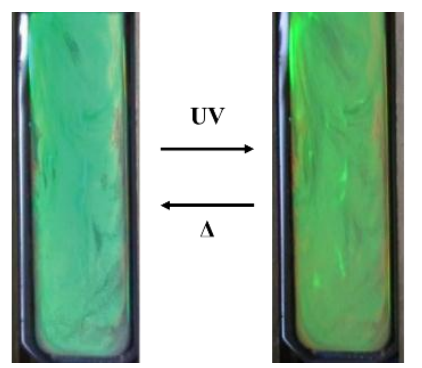

$[\mathrm{C18AA}]=40 \mathrm{mM}$

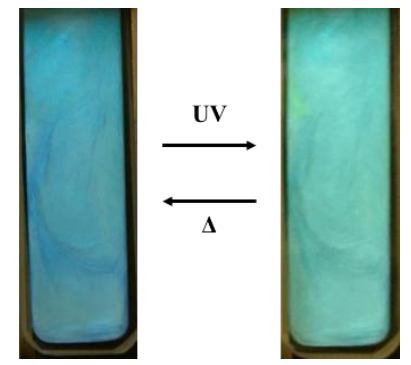

Figure 7. Color variation of $\mathrm{C} 18 \mathrm{AA}+\mathrm{TOAB}$ emulsion obtained by tuning the concentration of C18AA; $[\mathrm{TOAB}]=15 \mathrm{mM},[\mathrm{NaOH}]=3 \mathrm{mM}$, and $[$ Pyran $]=16 \mathrm{mM}$.

\section{Conclusions}

The addition of $\mathrm{NaOH}$ improved the brightness of the original $\mathrm{C} 18 \mathrm{AA}+\mathrm{TOAB}$ iridescent emulsion without affecting the coloring temperature and the lattice spacing of the iridescent emulsions. We also demonstrated that the addition of Pyran into C18AA + TOAB emulsions imparts dual thermo- and photo-responsive functions. The emulsion color was thermo- and photo-switchable between white and bluish green and between bluish green and green, respectively. Furthermore, the color can be switched between pink and pale orange or between blue and light blue by changing the original interference color of the emulsions via tuning the concentration of C18AA. The dual stimuli-responsive emulsions 
could be a possible as a one-chip sensor that can monitor both UV light and temperature. Further, if we can impart irreversibility of thermo- and photo-response into the iridescent emulsion, the emulsion is exploited as a smart sensor with the traceability of exposure to sunlight and heat.

Author Contributions: Conceptualization, T.K.; methodology, R.K.; formal analysis, all authors; writing-original draft preparation, T.K; writing-review and editing, all authors.

Funding: This research received no external funding.

Conflicts of Interest: The authors declare no conflicts of interest.

\section{References}

1. Kawai, T.; Hashizume, M. Stimuli-Responsive Interfaces; Springer: Singapore, 2017; ISBN 9789811024610.

2. Li, Q. Intelligent Stimuli-Responsive Materials: From Well-Defined Nanostructures to Applications; John Wiley\&Sons: Hoboken, NJ, USA, 2013; ISBN 9781118452004.

3. Urban, M.W. Stimuli-Responsive Materials: From Molecules to Nature Mimicking Materials Design; Royal Society of Chemistry: London, UK, 2016; ISBN 9781849736565.

4. Wang, L.; Li, Q. Photochromism into nanosystems: Towards lighting up the future nanoworld. Chem. Soc. Rev. 2018, 47, 1044-1097. [CrossRef] [PubMed]

5. Wang, L.; Urbas, A.M.; Li, Q. Nature-inspired emerging chiral liquid crystal nanostructures: from molecular self-assembly to dna mesophase and nanocolloids. Adv. Mater. 2018, 30, 1801335. [CrossRef] [PubMed]

6. Wang, L.; Li, Q. Stimuli-directing self-organized 3d liquid-crystalline nanostructures: from materials design to photonic applications. Adv. Funct. Mater. 2016, 26, 10-28. [CrossRef]

7. Weissman, J.M.; Sunkara, H.B.; Tse, A.S.; Asher, S.A. Thermally switchable periodicities and diffraction from mesoscopically ordered materials. Science 1996, 274, 959-960. [CrossRef] [PubMed]

8. Holtz, J.H.; Asher, S.A. Polymerized colloidal crystal hydrogel films as intelligent chemical sensing materials. Nature 1997, 389, 829-832. [CrossRef]

9. Takeoka, Y.; Watanabe, M. Tuning Structural Color Changes of Porous Thermosensitive Gels through Quantitative Adjustment of the Cross-Linker in Pre-gel Solutions. Langmuir 2003, 19, 9104-9106. [CrossRef]

10. Saito, H.; Takeoka, Y.; Watanabe, M. Simple and precision design of porous gel as a visible indicator for ionic species and concentration. Chem. Commun. 2003, 2126-2127. [CrossRef]

11. Schafer, C.G.; Gallei, M.; Zahn, J.T.; Engelhardt, J.; Hellmann, G.P.; Rehahn, M. Reversible Light-, Thermo-, and Mechano-Responsive Elastomeric Polymer Opal Films. Chem. Mater. 2013, 25, 2309-2318. [CrossRef]

12. Ito, T.; Katsura, C.; Sugimoto, H.; Nakanishi, E.; Inomata, K. Strain-Responsive Structural Colored Elastomers by Fixing Colloidal Crystal Assembly. Langmuir 2013, 29, 13951-13957. [CrossRef] [PubMed]

13. Shiraki, T.; Dawn, A.; Tsuchiya, Y.; Shinkai, S. Thermo- and Solvent-Responsive Polymer Complex Created from Supramolecular Complexation between a Helix-Forming Polysaccharide and a Cationic Polythiophene. J. Am. Chem. Soc. 2010, 132, 13928-13935. [CrossRef] [PubMed]

14. Gao, Y.; Serpe, M.J. Light-Induced Color Changes of Microgel-Based Etalons. ACS Appl. Mater. Interfaces 2014, 6, 8461-8466. [CrossRef] [PubMed]

15. Zhao, H.; Gao, J.; Pan, Z.; Huang, G.; Xu, X.; Song, Y.; Xue, R.; Hong, W.; Qiu, H. Chemically Responsive Polymer Inverse-Opal Photonic Crystal Films Created by a Self-Assembly Method. J. Phys. Chem. C 2016, 120, 11938-11946. [CrossRef]

16. He, Y.-D.; Zhang, Z.-L.; Xue, J.; Wang, X.-H.; Song, F.; Wang, X.-L.; Zhu, L.-L.; Wang, Y.-Z. Biomimetic Optical Cellulose Nanocrystal Films with Controllable Iridescent Color and Environmental Stimuli-Responsive Chromism. ACS Appl. Mater. Interfaces 2018, 10, 5805-5811. [CrossRef] [PubMed]

17. Tang, S.; Wang, C.; Liu, N.; Li, Y.; Han, P.; Lu, Q. Instantaneous Magnetically Assembled and Hydrophilic Photonic Crystals with Controlled Diffraction Colors. J. Phys. Chem. C 2018, 122, 18021-18028. [CrossRef]

18. Sato, E.; Masuda, Y.; Kadota, J.; Nishiyama, T.; Horibe, H. Dual stimuli-responsive homopolymers: Thermoand photo- responsive properties of coumarin-containing polymers in organic solvents. Eur. Polym. J. 2015, 69, 605-615. [CrossRef]

19. Tang, X.D.; Chen, Y.; Yu, F.Q.; Pei, M.S. Photo- and Thermo-Dual-Responsive Organic/Inorganic Hybrid Materials. Key Eng. Mater. 2013, 538, 181-184. [CrossRef] 
20. Morita, C.; Aoyama, T.; Imura, Y.; Kawai, T. Novel thermo-responsive coloring phenomena in water/surfactant/oil emulsions. Chem. Commun. 2011, 47, 11760-11762. [CrossRef] [PubMed]

21. Arai, K.; Kobayashi, Y.; Abe, J. Rational molecular designs for drastic acceleration of the color-fading speed of photochromic naphthopyrans. Chem. Commun. 2015, 51, 3057-3060. [CrossRef] [PubMed]

22. Morita, C.; Sugimoto, H.; Matsue, K.; Kondo, T.; Imura, Y.; Kawai, T. Change in Viscosity Behavior from a Normal Organogelator to a Heat-Induced Gelator for a Long-chain Amidoamine Derivative. Chem. Commun. 2010, 46, 7969-7971. [CrossRef] [PubMed]

23. Imura, Y.; Morita, C.; Sugimoto, H.; Kondo, T.; Kawai, T. Reversible Phase Transfer and Fractionation of Au Nanoparticles by pH Change. Chem. Commun. 2010, 46, 9206-9208. [CrossRef] [PubMed]

Sample Availability: Samples of the compounds are not available from the authors.

(C) 2018 by the authors. Licensee MDPI, Basel, Switzerland. This article is an open access article distributed under the terms and conditions of the Creative Commons Attribution (CC BY) license (http:/ / creativecommons.org/licenses/by/4.0/). 